\title{
Research on Management Strategy of Cost-based Intelligent Manufacturing System
}

\author{
Cong Haoting ${ }^{1}$, Che Jinyi ${ }^{2}$ \\ ${ }^{1}$ Ocean University of China, University of Waterloo
}

\begin{abstract}
[Abstract] The reliability, efficiency and accuracy of CNC machines as work cells of intelligent manufacturing systems (IMS) are criteria to measure the processing level of the latter. In order to improve the reliability of the IMS and reduce the maintenance cost, very sound preventive maintenance and management strategies concerning the $\mathrm{CNC}$ machines should be defined. We realized a parameter estimation of our reliability model for CNC machine units in an IMS environment, carried out a linear correlation test and a distribution fitting test for the model and obtained the failure distribution function and failure rate function. We then built a post-failure maintainability model and realized a maintainability evaluation. Following the above analyses, we built a cost-based preventive maintenance cycle model and obtained its optimal value by using the particle swarm optimization (PSO) algorithm. This research and its result can on the one hand guide the setting-up of preventive maintenance planning and management schemes and on the other hand reduce the production cost and enhance enterprise efficiency.
\end{abstract}

\section{CLC classification number : TG659 Documentary ID code : A}

\section{Preface}

Over maintenance and lack of maintenance are two common problems that exist in IMS maintenance processes. Excessive preventive maintenance leads to increase of total maintenance cost while lack of preventive maintenance often causes more frequent machine failures and thus increases maintenance cost. The maintenance cost control and the optimal maintenance efficiency demand that the preventive maintenance cycle be soundly defined and the balance be found between the failure time and checking time in order to reduce the maintenance cost per unit time. It is therefore imperative to research on the preventive maintenance and its management strategies [1-4].

Scholars of the field have carried out a large number of studies. Xiaofeng Wang et al built an equipment reliability model by using the three-parameter Weibull distribution, taking into consideration the preventive maintenance (PM) cost and post maintenance cost caused by accidental failures. They then underwent its parameter optimization by differential evolution algorithm. They also carried out researches on two PM strategies on multi-device series systems [5].

Tantele developed an optimization methodology based on genetic algorithm (GA) principles and Bayesian updating[6]. Abubaker proposed a maintenance cost estimation model within the research and development of this decision support system (DSS). An

15563100128@163.com,j7che@uwaterloo.ca empirical-based methodology is pursued and validated through case study analysis [7].

Jiang et al, through their analysis of the inertial navigation system and the redundant system of the equipment studied, established the objective/goal function that aimed at the minimalization of the maintenance cost and obtained the equipment's PM cycle. The present research is based on our tracking records of failure time of a machining center over a span of months. The detailed data have permitted us to realize a parameter estimation, a linear correlation test and a distribution fitting test of our reliability model. They have further enabled us to build a model for the post-failure maintainability as well as to carry out its evaluation. In the end, we built a model for the CNC machine's cost-based preventive maintenance cycle, and obtained its optimal value by using the particle swarm optimization (PSO) algorithm.

\section{Reliability Model}

We built a reliability model with the failure data of a machining center. Among the data, 29 failure intervals are randomly selected, as shown in table 1 .

Table 1 Failure time of tool magazine

\begin{tabular}{cccccc}
\hline$i$ & $t_{i}(h)$ & $i$ & $t_{i}(h)$ & $i$ & $t_{i}(h)$ \\
\hline 1 & 12 & 11 & 211 & 21 & 394 \\
2 & 45 & 12 & 234 & 22 & 417 \\
\hline
\end{tabular}




\begin{tabular}{cccccc}
\hline 3 & 54 & 13 & 237 & 23 & 557 \\
4 & 54 & 14 & 259 & 24 & 565 \\
5 & 61 & 15 & 266 & 25 & 591 \\
6 & 63 & 16 & 271 & 26 & 729 \\
7 & 70 & 17 & 274 & 27 & 769 \\
8 & 92 & 18 & 296 & 28 & 944 \\
9 & 96 & 19 & 346 & 29 & 1044 \\
10 & 104 & 20 & 372 & & \\
\hline
\end{tabular}

\subsection{Parameter Estimation of the Reliability Model}

According to previous reliability studies on machining centers, the cumulative failure distribution function abides by the Weibull distribution [15]. The two-parameter Weibull distribution function is :

$$
F(t)=1-\exp \left[-\left(\frac{t}{\alpha}\right)^{\beta}\right], \quad t \geq 0
$$

The corresponding reliability function is :

$$
R(t)=\exp \left[-\left(\frac{t}{\alpha}\right)^{\beta}\right], \quad t \geq 0
$$

Let the univariate linear regression equation be

$$
y=A+B x
$$

According to the two-parameter Weibull distribution, formula (1) can be linearly transformed as following:

:

$$
y_{i}=\ln \ln \frac{1}{1-F\left(t_{i}\right)}, x_{i}=\ln t_{i}, A=-\beta \ln \alpha, B=\beta
$$

The value $F(t)$ needs to be estimated before calculation. In general, the median rank is used to estimate $\mathrm{F}(\mathrm{t})$, that is :

$$
\hat{F}\left(t_{i}\right) \approx \frac{i-0.3}{n+0.4}
$$

According to the least square method, the parameter estimation is drawn as below:

$$
\begin{gathered}
\hat{B}=\frac{\sum_{i=1}^{n} x_{i} y_{i}-\frac{1}{n} \sum_{i=1}^{n} x_{i} \cdot \sum_{i=1}^{n} y_{i}}{\sum_{i=1}^{n} x_{i}^{2}-\frac{1}{n}\left(\sum_{i=1}^{n} x_{i}\right)^{2}} \\
\hat{A}=\frac{1}{n} \sum_{i=1}^{n} y_{i}-\hat{B} \cdot \frac{1}{n} \sum_{i=1}^{n} x_{i}
\end{gathered}
$$

At last we can get that:

$$
\hat{\beta}=\hat{B}, \hat{\alpha}=\exp (-\hat{A} / \hat{B}) \text { 。 }
$$

In line with the discussion above and with the cumulative failure time data of the headstock (refer to Table 1 ), the headstock reliability model parameter was obtained as: $\beta=1.0805, \alpha=345.5$ 。

\subsection{Hypothesis Test of the Reliability Model}

\subsubsection{Linear Correlation Test}

The linear correlation coefficient is:

$$
\hat{\rho}=\frac{\sum_{i=1}^{n} x_{i} y_{i}-\frac{1}{n} \sum_{i=1}^{n} x_{i} \cdot \sum_{i=1}^{n} y_{i}}{\sqrt{\left[\sum_{i=1}^{n} x_{i}^{2}-\frac{1}{n}\left(\sum_{i=1}^{n} x_{i}\right)^{2}\right]\left[\sum_{i=1}^{n} y_{i}^{2}-\frac{1}{n}\left(\sum_{i=1}^{n} y_{i}\right)^{2}\right]}}
$$

When $|\hat{\rho}|_{>} \rho_{(n-2, \alpha)}$, we consider that the linear correlation between $\mathrm{X}$ and $\mathrm{Y}$ is significant. $\rho_{(n-2, \alpha)}$, the critical value of the correlation coefficient $\rho$, can be obtained either by consulting the table or by approximate formula calculation. The latter method is adopted in this paper, the significance level being $\alpha=0.1$. Therefore,

$$
\rho_{(n-2, \alpha)}=\frac{1.645}{\sqrt{v+1}}
$$

In the formula, $v=n-2, \mathrm{n}$ is the cumulative failure number.Using the correlation coefficient method, we carried out a hypothesis test on the failure distribution of the headstock. Formula (4) revealed that $\hat{\rho}=0.9833$, and $\rho_{(n-2 v)}=0.3226$. Thus, $\hat{\rho}>\rho_{(n-2 v)}, \quad$ and accordingly, the linear correlation between $\mathrm{X}$ and $\mathrm{Y}$ is significant. Therefore, the failure distribution of the headstock's subsystem obeys the Weibull distribution theory.

\subsubsection{Hypothesis Test of the Fitting of Distribution}

Using the "d" test method, we checked the failure time distribution function. If the distribution function obtained from the estimated parameter meet the following conditions, the parameter estimation is well founded. Suppose that:

$$
D_{n}=\sup _{-\infty<x<+\infty}\left|F_{n}(x)-F_{0}(x)\right|=\max \left\{d_{i}\right\} \leq D_{n, \alpha}
$$

In the formula, $\mathrm{F}_{0}(\mathrm{x})$ is the original hypothetical distribution function, $\mathrm{F}_{\mathrm{n}}(\mathrm{x})$ is the empirical distribution function with the sample size being $\mathrm{n}$, and $D_{n, \alpha}$ is the critical value.

For $d_{i}$, details are as follows:

$$
d_{i}=\max \left\{F_{0}\left(x_{i}\right)-\frac{i-1}{n}, \frac{i}{n}-F_{0}\left(x_{i}\right)\right\}
$$

After carrying out a "d" test on the earlier calculated headstock failure distribution function.

$$
F(t)=1-\exp \left[-\left(\frac{t}{2025.350}\right)^{1.239}\right]
$$

We obtained the value $D_{n}=0.106$. When the significance level is $0.1, D_{n, \alpha}=0.3026$. Thus the result $D_{n} \leq D_{n, a}$ complies with the hypothesis test. The above demonstration then made it possible to calculate the failure distribution function, failure rate function and probability density function for each component of the machining center, as shown in Table 2. 
Table 2. Reliability function

\begin{tabular}{cc}
\hline & 可靠性函数 Reliability function \\
\hline $\begin{array}{c}\text { 故障分布函数 } \\
\text { Failure distribution function }\end{array}$ & $F(t)=1-\exp \left[-(t / 345.5)^{1.0805}\right]$ \\
$\begin{array}{c}\text { 概率密度函数 } \\
\text { Probability density function } \\
\text { 故障率函数 } \\
\text { Failure rate function }\end{array}$ & $f(t)=\frac{1.0805}{345.5}\left(\frac{t}{345.5}\right)^{0.0805} \exp \left[-\left(\frac{t}{345.5}\right)^{1.0805}\right]$ \\
& $\lambda(t)=\frac{1.0805}{345.5}\left(\frac{t}{345.5}\right)^{0.0805}$ \\
\hline
\end{tabular}

\section{Maintainability Model Analysis}

\subsection{Maintenance Model Establishment}

The post-failure maintenance of the machine tools demands research on their maintainability.

Therefore, the building-up of maintainability model not only is the key to quantitative research on system maintainability but also lays the foundation for further research on maintenance cost.

Table 3 shows the post-failure maintenance time data recorded during field tests at a series machining center.

Table 3. Troubleshooting time-taken list

\begin{tabular}{llll}
\hline Group No. & Time-taken & $\begin{array}{l}\text { Number of } \\
\text { breakdown }\end{array}$ & Frequency \\
\hline 1 & 0.5 & 10 & 0.3448 \\
2 & 1 & 7 & 0.24137 \\
3 & 1.5 & 6 & 0.20689 \\
4 & 2 & 3 & 0.103448 \\
5 & 3.5 & 1 & 0.03448 \\
6 & 5.5 & 1 & 0.03448 \\
7 & 7.5 & 1 & 0.03448 \\
\hline
\end{tabular}

According to experience, the distribution type of the failure repairing time conforms to lognormal distribution, the probability density function of the latter being :

$$
m(t)=\frac{1}{\sigma t \sqrt{2 \pi}}^{-\frac{1}{2}\left(\frac{\ln t-\mu}{\sigma}\right)^{2}}
$$

In the formula, $\mu_{\text {is }} \ln t$ 's mean value and $\sigma$ is $\ln t$ 's variance.

The lognormal cumulative distribution function is the following:

$$
M(t)=\int_{0}^{t} \frac{1}{\sigma x \sqrt{2 \pi}}^{-\frac{1}{2}\left(\frac{\ln x-\mu}{\sigma}\right)^{2}} d x
$$

We used the maximum likelihood method to estimate its parameters. By assuming that the observed maintenance time at the machining center $t_{1}, t_{2}, \cdots t_{n}$ was a sample of a lognormal distribution population, we obtained the likelihood function as follows:

$$
L(t ; \mu, \sigma)=\prod_{i=1}^{n} \frac{1}{\sigma t_{i} \sqrt{2 \pi}} \exp \left(-\frac{1}{2}\left(\frac{\ln t_{i}-\mu}{\sigma}\right)^{2}\right)
$$

To take logarithm for the above formula, the corresponding maximum likelihood equation is:

$$
\left\{\begin{array}{l}
\frac{\partial \ln L(t ; \mu, \sigma)}{\partial \mu}=\frac{1}{2 \sigma^{2}} \sum_{i=1}^{n} 2\left(\ln t_{i}-\mu\right)=0 \\
\frac{\partial \ln L(t ; \mu, \sigma)}{\partial \sigma^{2}}=-\frac{n}{2} \frac{2 \pi}{2 \pi \sigma^{2}}+\frac{1}{2 \sigma^{4}} \sum_{i=1}^{n}\left(\ln t_{i}-\mu\right)^{2}=0
\end{array}\right.
$$

The parameter estimation for lognormal distribution was :

$$
\left\{\begin{array}{l}
\hat{\mu}=\frac{1}{n} \sum_{i=1}^{n} \ln t_{i} \\
\hat{\sigma}^{2}=\frac{1}{n} \cdot \sum_{i=1}^{n}\left(\ln t_{i}-\hat{\mu}\right)^{2}
\end{array}\right.
$$

Under the premise that the failure repairing time at the machining center obeyed the lognormal distribution, we calculated the parameter estimates:

$\hat{\mu}=0.088, \quad \hat{\sigma}=0.732$ 。

Thus the maintainability function $M(t)$ was reached at as below:

$$
M(t)=\int_{0}^{t} \frac{1}{0.732 x \sqrt{2 \pi}} \cdot e^{-\frac{1}{2}\left(\frac{\ln x-0.088}{0.732}\right)^{2}} \cdot d x
$$

\subsection{Maintainability Evaluation}

Mean Time To Repair (MTTR)

The estimated MTTR value was calculated by the formula:

$$
\text { MTTR }=\int_{0}^{\infty} t m(t) d t=\exp \left(\mu+\frac{\sigma^{2}}{2}\right)
$$

Thus we obtained the mean time taken for repair: $\mathrm{MTTR}=1.427 \mathrm{~h}$.

\section{Preventive Maintenance Cycle Model}

\subsection{Preventive Maintenance Cycle Model for Machine Tools}

In order to build the preventive maintenance cycle model based on failure rates, we prescribed the following assumptions: 
1. After each preventive maintenance, the failure rate of the machine tool returns to original level

2. When the machine tool breaks down within a PM cycle, it will be repaired. Such maintenance can restore its function but cannot affect its failure rate after repairing.

The formula of the preventive maintenance cycle model which is based on failure rates is:

$$
C(T)=\frac{c_{f} d_{f} \int_{0}^{T} \lambda(t) d t+c_{p} d_{p}+\left[d_{f} \int_{0}^{T} \lambda(t) d t+d_{p}\right] S}{T}
$$

The preventive maintenance cycle being $\mathrm{T}, \mathrm{C}(\mathrm{T})$ refers to the expected total maintenance cost per unit time. $\lambda(\mathrm{t})$ is the machine tool's failure rate, $d_{f}$ is its mean failure-induced downtime. Therefore, $d_{f}=$ MTTR . $\int_{0}^{T} \lambda(t) d t$ refers to the number of failures occurring within the preventive interval cycle $\mathrm{T}, c_{f}$ to the maintenance cost per unit time during the failure period, $c_{p}$ to the cost for preventive maintenance per unit time, $d_{p}$ to the average downtime value for preventive maintenance, and $\mathrm{S}$ to the cost loss per unit time due to machine breakdown.

Assuming on the one hand that $\mathrm{N}$ failures occurred to a machine tool, each of which demanded the repair time $d_{f}(i)$ and the repair cost $c_{f}(i)$, in which $i=1,2, \ldots \mathrm{N}$, and on the other hand $\mathrm{M}$ preventive maintenances were carried out on the machine tool, each of which demanded the repair time $d_{p}(j)$ and repair cost $c_{p}(j)$, in which $j=1,2, \ldots \mathrm{N}$, we obtained the following formula:

$$
\begin{aligned}
& \left\{\begin{array}{l}
d_{f}=\text { MTTR } \\
c_{f}=\sum_{i=1}^{N} c_{f}(i) /\left(N \cdot d_{f}\right)
\end{array}\right. \\
& \left\{\begin{array}{l}
d_{p}=\sum_{j=1}^{M} d_{p}(j) / M \\
c_{p}=\sum_{j=1}^{M} c_{p}(j) /\left(M \cdot d_{p}\right)
\end{array}\right.
\end{aligned}
$$

\subsection{Preventive Maintenance Model: Calculation and Analysis}

The above demonstration delivered the result $d_{f}=M T T R=1.427$ and according to the data provided by the machine owner, $c_{f}=600, c_{p}=200, d_{p}=2, \mathrm{~S}=100$. The failure rate function $\lambda(t)$ could then be calculated by following Table 2 and the expression of $\mathrm{C}(\mathrm{T})$ could be determined through the formula 14 .

$C(T)=\frac{c_{f} d_{f} \int_{0}^{T} \lambda(t) d t+c_{p} d_{p}+\left[d_{f} \int_{0}^{T} \lambda(t) d t+d_{p}\right] S}{T}$

$$
\begin{gathered}
=\frac{600 \times 1.427 \times \int_{0}^{T} \frac{1.0805}{345.5}\left(\frac{t}{345.5}\right)^{0.0805} d t+200 \times 2}{T} \\
+\frac{\left[1.427 \times \int_{0}^{T} \frac{1.0805}{345.5}\left(\frac{t}{345.5}\right)^{0.0805} d t+2\right] \times 100}{T} \\
=\frac{1.806 \times T^{1.0805}+598.2}{T}
\end{gathered}
$$

\subsection{Parameter Optimization based on Particle Swarm Optimization Algorithm}

The theory of particle swarm optimization (PSO) is derived from research on birds' predation behavior. The algorithm is characteristically easy to implement, highly precise and of high convergence speed. It has shown its advantages in solving practical problems in many fields. We used PSO algorithm to optimize the expression of $\mathrm{C}(\mathrm{T})$, i.e. formula (17). Figure 1 demonstrates the PSO parameter optimization flow:

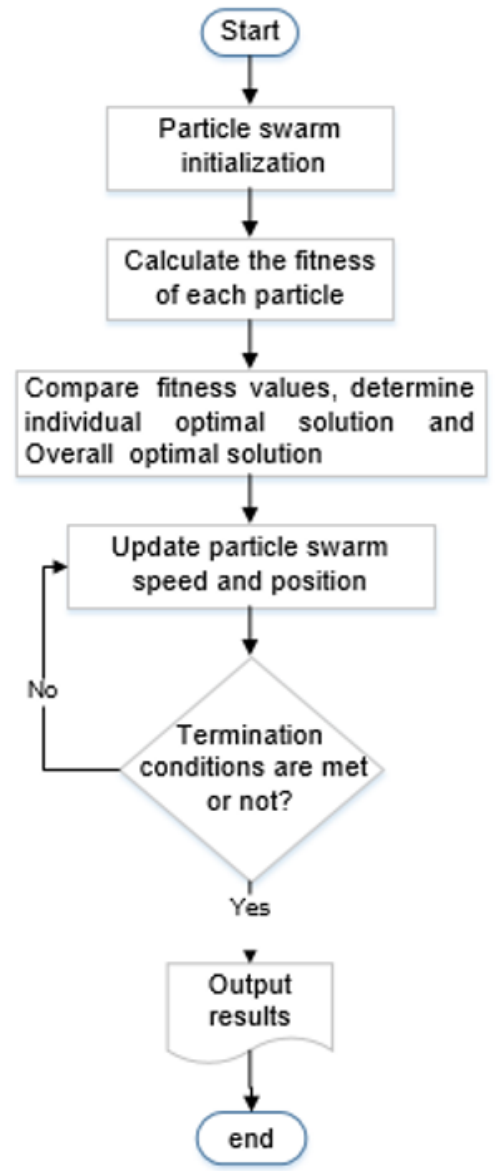

Figure 1. PSO Parameter Estimation Flow Chart

After the optimization of the $\mathrm{C}(\mathrm{T})$ with $\mathrm{PSO}$, we found that when $\mathrm{T}=2213$, the corresponding total maintenance cost $\mathrm{C}(\mathrm{T})$ per unit time reached its minimum value, 


$$
C_{\min }(T)=C(2213)=\mathbf{3 . 6 2 7 6 8 4 1} \text { 。 }
$$

We also got the correlation between the total maintenance cost $\mathrm{C}(\mathrm{T})$ per unit time and the preventive maintenance cycle, as shown in Figure 2. As the cycle $\mathrm{T}$ increases, the $\mathrm{C}$ value decreases. When $\mathrm{T}=2213 \mathrm{~h}$, maintenance cost per unit time reaches its minimum value and then gradually increases following the increase of time value.

Therefore, reliable forecast of the preventive maintenance model and optimal maintenance and management of the machining tool can reduce the maintenance cost and enhance the productivity of the enterprise.

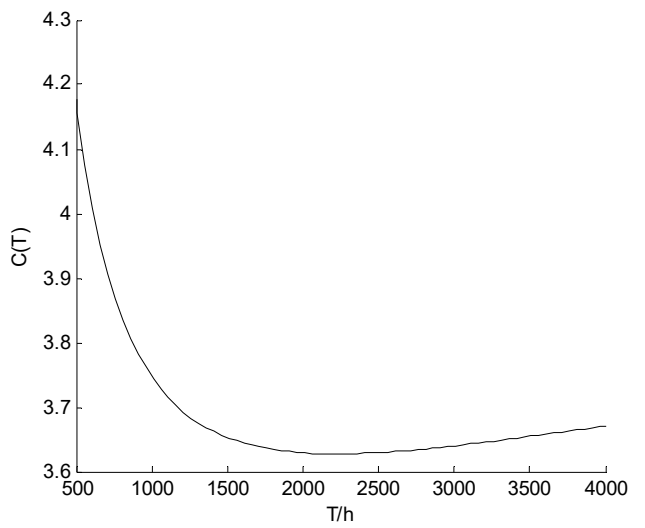

Figure 2. Correlation Between Maintenance Cost and Maintenance Cycle per Unit Time

\section{Conclusion}

In order to improve the reliability and reduce the maintenance cost of the intelligent manufacturing system, it is crucial to formulate sound preventive maintenance and management strategies for the CNC machine tools. In this research we have realized estimates on the reliability model's parameters of a CNC machine tool in an IMS, and carried out a linear correlation test and a distribution fitting test on the model. We have thus obtained the failure distribution function and the failure rate function. Through this case study.the methodology for model building-up and relevant maintainability evaluation is available and instructive for readers' reference.

In addition to these analyses, we have built a failure rate based preventive maintenance cycle model and optimized it with the particle swarm optimization (PSO) algorithm until it reached its optimal value. The cost-based research methods adopted in this paper and the conclusions it has reached can help IMS machine management teams set up well founded preventive maintenance planning and management schemes in order to reduce the production cost and enhance enterprise efficiency.

\section{Reference}

1. Zahid Hussain, Hamid Jan. Establishing simulation model for optimizing efficiency of CNC machine using reliability-centered maintenance approach.
2019, 10(06)

2. Hongyan Dui,Shubin Si,Richard C.M. Yam. A cost-based integrated importance measure of system components for preventive maintenance[J]. Reliability Engineering and System Safety,2017.

3. Aghezzaf E H , Khatab A, Tam P L . Optimizing production and imperfect preventive maintenance planning's integration in failure-prone manufacturing systems[J]. Reliability Engineering and System Safety, 2016, 145:1-8.

4. Chalabi N, Dahane M , Beldjilali B , et al. Optimisation of preventive maintenance grouping strategy for multi-component series systems: Particle swarm based approach[J]. Computers \& Industrial Engineering, 2016, 102:440-451.

5. Xiaofeng Wang,Shu Guo,Jian Shen,Yang Liu. Optimization of preventive maintenance for series manufacturing system by differential evolution algorithm[J]. Journal of Intelligent Manufacturing,2020,31(3).

6. E. A. Tantele,T. Onoufriou. Optimum preventative maintenance strategies using genetic algorithms and Bayesian updating $[\mathrm{J}]$. Ships and Offshore Structures, 2009,4(3).

7. Abubaker, Shagluf, Simon, et al. Adaptive decision support for suggesting a machine tool maintenance strategy From reactive to preventative[J]. Journal of Quality in Maintenance Engineering, 2018.

8. Jiang X, Duan F, Tian H, et al. Optimization of reliability centered predictive maintenance scheme for inertial navigation system[J]. Reliability Engineering \& System Safety, 2015, 140: 208-217 